\title{
Letters to the
}

\section{Editor}

The Editor welcomes submissions for possible publication in the Letters to the Editor section that consist of commentary on an article published in the Journal or other relevant issues. Authors should:

- Include no more than 500 words of text, three authors, and five references

- Type with double-spacing

- See http://jtcs.ctsnetjournals.org/ misc/ifora.shtml for detailed submission instructions.

- Submit the letter electronically via jtcvs.editorialmanager.com.

Letters commenting on an article published in the JTCVS will be considered if they are received within 6 weeks of the time the article was published. Authors of the article being commented on will be given an opportunity of offer a timely response ( 2 weeks) to the letter. Authors of letters will be notified that the letter has been received. Unpublished letters cannot be returned.

\section{Management of spinal cord perfusion pressure to minimize intermediate-delayed paraplegia: Critical role of central venous pressure \\ To the Editor:}

I read with great interest the recent article by Etz and colleagues ${ }^{1}$ detailing their extensive experience in thoracoabdominal aortic aneurysm repair (858 cases: 19902006). The authors have clearly demonstrated the significant relationship between relative hypotension (mean arterial pressure $80 \%$ of preoperative baseline) and the risk of perioperative spinal cord ischemia $(P=.027)$. Furthermore, they also detected a significant association between an elevated postoperative central venous pressure and the risk of spinal cord ischemia $(P=.03)$.

The following questions arise as a consequence of this fascinating study:

1. Should we define spinal cord perfusion pressure as follows: (mean arterial pressure - central venous pressure) - cerebrospinal fluid pressure?

On the basis of the data presented by the authors and our current understanding of spinal cord physiology, this definition makes sense. Traditionally, central venous pressure has been omitted, because it has generally not been considered clinically significant in this setting.

2. If this definition is acceptable, does it suggest a more rational approach to the optimization of spinal cord perfusion pressure by manipulation of each parameter?

3. What central venous pressure would the authors suggest as a goal during corrective management for intermediate-delayed paraplegia?

4. What pharmacologic management would the authors suggest or consider optimal to increase mean arterial pressure and lower central venous pressure?

I congratulate the authors again on their important contribution. I look forward to their comments about this aspect of paraplegia management after thoracoabdominal aortic aneurysm repair.
John G. T. Augoustides, MD, FASE Department of Anesthesiology and Critical Care Hospital of the University of Pennsylvania

Philadelphia, $\mathrm{Pa}$

Financial Support: Department of Anesthesiology and Critical Care, Hospital of the University of Pennsylvania.

\section{Reference}

1. Etz CD, Luehr M, Kari FA, Bodian CA, Smego D, Plestis KA, et al. Paraplegia after extensive thoracoabdominal aortic aneurysm repair: does critical spinal cord ischemia occur postoperatively? J Thorac Cardiovasc Surg. 2008;135:324-30.

doi:10.1016/j.jtcvs.2008.02.075

\section{Reply to the Editor:}

We thank Dr Augoustides for his interest and questions.

It seems to us indisputable that ADEQUATE spinal cord perfusion during the first 24 hours postoperatively is critical for the prevention of intermediate delayed paraplegia after thoracic and thoracoabdominal aortic aneurysm repair. Our study made the relatively new observation that a normal blood pressure, if below a patient's preoperative level, can be a risk factor for intermediate delayed paraplegia. This buttresses the argument, which has been made for some time, that higher-than-normal arterial pressures may be necessary for adequate perfusion in the immediate postoperative period for patients after resection of descending thoracic and thoracoabdominal aneurysms to prevent paraplegia because antecedent pressure in many of these patients has been higher than normal. The current observation-linking the required pressure to the patient's presenting blood pressure-now suggests that target arterial pressures during the early postoperative period should be individualized.

Similarly, it has been known for some time that cerebrospinal fluid (CSF) pressure is an important component of spinal cord perfusion pressure (SCPP), and that CSF drainage helps to prevent spinal cord injury. What is relatively new is the 
idea that, especially in the absence of a measurement of CSF pressure (ie, in those patients without spinal cord drainage), central venous pressure (CVP) can perhaps be used as a substitute, in this context, for CSF pressure. We think it is unlikely that CSF pressure can ever be less than CVP, but the exact relationship between them may not be entirely straightforward despite the observation that CSF and CVP correlate closely during normal cardiopulmonary circulation. ${ }^{1,2}$ The relevance of a high CVP in the context of spinal cord perfusion is explained by the fact that the arterial pressure of the inflow to the spinal cord after segmental artery sacrifice is only a fraction of systemic mean arterial pressure (MAP), as explained next.

We think that establishing an index for assessing the adequacy of the postoperative SCPP is an excellent idea. However, among the parameters readily available in the clinical setting (in the operating room and intensive care unit), we believe the most important variable to adequately assess SCPP is missing: the actual inflow arterial pressure in the collateral system supplying the most vulnerable region of the spinal cord. To obtain this pressure, we have recently adapted a method we developed in our laboratory: we insert a small catheter directly into the stump of a thoracic or lumbar segmental artery during surgery to measure the pressure in the collateral circulation during the operation, and for a variable interval postoperatively when possible.

After extensive segmental artery occlusion, when inflow is provided predominantly by the collateral network, the arterial input for SCPP routinely decreases to levels as low as $20 \%$ of MAP, according to our experimental studies. ${ }^{3,4}$ We have confirmed similarly low levels of inflow SCPP (20\% of MAP) transiently in patients after segmental artery sacrifice using a catheter placed in L1. In patients with an L1 catheter and CSF drainage, an accurate SCPP can be calculated as SCPP $=\mathrm{L} 1$ pressure - CSF pressure.

The acceptable minimum SCPP-the value recorded at the end of operation in the presence of intact function as documented by SSEP/MEP monitoring - could be used as a baseline when calculating an adequate flow for postoperative follow-up: SCPP index $=$ current $\mathrm{SCPP}-$ minimum SCPP.

Such an SCPP index could be monitored along with the usual vital signs, and changes in hemodynamics instituted if it de- creases below zero. A negative SCPP index would indicate that spinal cord perfusion is below safe levels and trigger corrective measures, such as increasing MAP and decreasing CSF or CVP to improve perfusion; the target SCPP index should be zero or more at all times. An additional CONSIDERATION is that the patient may warm significantly after surgery, and increases in metabolic rate in the cord, as well as other tissues supplied by the collateral flow, THAN AT BASELINE. For calculation of A SCPP index in the absence of CSF pressure measurements, CVP could be substituted.

In the absence of direct monitoring, however, we think that routine consideration of AN INDIVIDUAL'S preoperative MAP would be a simplified way for personnel charged with the care of patients after aneurysm surgery which poses a risk of paraplegia to monitor the minimum systemic pressures necessary to provide adequate spinal cord perfusion without their being required to have a sophisticated understanding of spinal cord perfusion requirements. In general, we think that a CVP less than $10 \mathrm{~mm} \mathrm{Hg}$ would be acceptable, but a high CVP may have to be treated more aggressively if arterial pressure is low. To raise the arterial pressure, we would - in view of the need to keep the CVP low-advocate the use of pharmacologic agents such as epinephrine or norepinephrine rather than using volume infusions.

We thank Dr Augoustides for raising these issues and the editorial board for the opportunity of discussing them.

Christian D. Etz, MD

Randall B. Griepp, MD Mount Sinai School of Medicine New York, NY

\section{References}

1. Grum DF, Svensson LG. Changes in cerebrospinal fluid pressure and spinal cord perfusion pressure prior to cross-clamping of the thoracic aorta in humans. J Cardiothorac Vasc Anesth. 1991;5:331-6.

2. Huynh TT, Miller CC 3rd, Estrera AL, et al. Correlations of cerebrospinal fluid pressure with hemodynamic parameters during thoracoabdominal aortic aneurysm repair. Ann Vasc Surg. 2005;19:619-24.

3. Etz CD, Homann TM, Plestis KA, et al. Spinal cord perfusion after extensive segmental artery sacrifice: can paraplegia be prevented? Eur J Cardiothorac Surg. 2007;31:643-8.
4. Etz CD, Homann TM, Luehr M, et al. Spinal cord blood flow and ischemic injury after experimental sacrifice of thoracic and abdominal segmental arteries. Eur J Cardiothorac Surg. 2008;33:1030-8. Epub 2008 Apr 11.

doi:10.1016/j.jtcvs.2008.05.008

\section{Cardiac stem cells in the real world}

\section{To the Editor:}

We read with great interest the recent article by Pouly and colleagues. ${ }^{1}$ These authors suggest that c-kit ${ }^{+}$cells retrieved out of human endomyocardial biopsies do not express the classic markers of stemness but are actually mast cells. By immunofluorescent staining on paraffin slices they show that $\mathrm{c}-\mathrm{kit}^{+}$cells are $\mathrm{CD} 45^{+}$and $\mathrm{CD} 105^{-}$. Furthermore they found that these c-kit ${ }^{+}$ cells also coexpress tryptase, a granule-derived serine proteinase, and as a consequence appear to be mast cells.

In our experiments, right atrial appendages were removed during routine cardiac surgery and cultured as previously described. ${ }^{2}$ Immunofluorescent staining was performed on cells growing out of the explants after 2 weeks. Primary labeled antibodies directed against c-kit (phycoerythrin), CD45 (fluorescein isothiocyanate [FITC]), and CD105 (FITC) were used. A small percentage of CD $45^{+}$cells could be detected. These CD $45^{+}$cells did not stain positive for c-kit (Figure 1, $A$ ). In contrast, a subpopulation of $\mathrm{CD} 45^{-}$cells expressed c-kit (Figure 1, A). This is in contradiction with the results published by Pouly and colleagues. ${ }^{1}$ Double staining with CD105 (FITC) and c-kit (phycoerythrin) revealed that all cells in the outgrowth are $\mathrm{CD} 105^{+}$and that a subpopulation of these cells coexpress c-kit (Figure 1, B). These findings refute that c-kit ${ }^{+}$cells would be mast cells $\left(\mathrm{CD} 45^{+}\right.$, tryptase ${ }^{+}, \mathrm{CD}_{105^{-}}$, and c$\mathrm{kit}^{+}$). Our findings are in accordance with those published by Smith and colleagues ${ }^{2}$ and Bearzi and colleagues. ${ }^{3}$ Their immunofluorescence and flow cytometric analysis also show that $\mathrm{c}-\mathrm{kit}^{+}$cells are $\mathrm{CD} 45^{-}$but do express CD105.

Pouly and colleagues ${ }^{1}$ state that care has to be taken that phenotypic changes could have occurred by passaging the cells. According to the authors, this could explain why differences in the antigen expressing profile are detected. In our setting, cells were not passaged or processed; immunofluorescence was performed directly on the cell 